Journal of

\section{Applied Crystallography}

ISSN 0021-8898

Received 18 September 2007

Accepted 10 January 2008

\title{
Simulating X-ray diffraction of textured films
}

\author{
Dag W. Breiby, ${ }^{\text {a* }}$ Oliver Bunk, ${ }^{\mathrm{b}}$ Jens W. Andreasen, ${ }^{\mathrm{c}}$ Henrik T. Lemke ${ }^{\mathrm{d}}$ and \\ Martin M. Nielsen ${ }^{\mathrm{c}, \mathrm{d}}$
}

\begin{abstract}
a Department of Physics, Norwegian University of Science and Technology, Høgskoleringen 5, 7491 Trondheim, Norway, 'b Paul Scherrer Institut, 5232 Villigen PSI, Switzerland, ' $P$ Polymer Department, Risø National Laboratory, PO Box 49, Frederiksborgvej 399, 4000 Roskilde, Denmark, and ${ }^{\text {d Danish }}$ National Research Foundation Centre for Molecular Movies, Niels Bohr Institute, University of Copenhagen, Universitetsparken 5, 2100 Copenhagen $\varnothing$, Denmark. Correspondence e-mail: dag.breiby@ntnu.no
\end{abstract}

\begin{abstract}
Computationally efficient simulations of grazing-incidence X-ray diffraction (GIXD) are discussed, with particular attention given to textured thin polycrystalline films on supporting substrates. A computer program has been developed for simulating scattering from thin films exhibiting varying degrees of preferred orientation. One emphasized common case is that of a 'fibre' symmetry axis perpendicular to the sample plane, resulting from crystallites having one well defined crystal facet towards the substrate, but no preferred inplane orientation. Peak splitting caused by additional scattering from the totally substrate-reflected beam (two-beam approximation) and refraction effects are also included in the program, together with the geometrical intensity corrections associated with GIXD measurements. To achieve 'user friendliness' for scientists less familiar with diffraction, the mathematically simplest possible descriptions are sought whenever feasible. The practical use of the program is demonstrated for a selected thin-film example, perylene, which is of relevance for organic electronics.
\end{abstract}

(C) 2008 International Union of Crystallography Printed in Singapore - all rights reserved

\section{Introduction}

X-ray diffraction is the most important technique for investigations of periodic structures at the nano-scale. Despite the steady progress of scanning probe techniques, there is a wealth of information that can exclusively or most conveniently be obtained by diffraction. This includes the unit-cell parameters, crystalline structure, crystallinity and sample anisotropy. For instance, in the rapidly evolving field of organic electronics, future success depends crucially on the ability to characterize molecular structures in organic thin films (Sirringhaus et al., 1999; Kuzmenko et al., 2001; Samuelsen et al., 2003; Breiby et al., 2005). Whereas a single crystal exhibits a 'perfect' threedimensional lattice, powders and films tend to exhibit varying degrees of order and preferred orientation. Powder and single-crystal diffraction techniques have reached an impressive degree of sophistication and automation during recent years, with a corresponding rich amount of literature and available software. Computer tools for analysing the inbetween cases, ranging from oriented powders to nonperfect single crystals, have proven more difficult to develop. Apart from the computer programs $R O D$ (Vlieg, 2000) for optimization of surface structures and Is GISAXS (Lazzari, 2002) for grazing-incidence small-angle scattering, no simulation programs are available, in particular not for grazing-incidence wide-angle scattering.

In the common case of a sample with crystallites having a (high) degree of (uniaxial) preferred orientation about one axis, an intermediate case of a 'two-dimensional powder' with cylindrical symmetry exists. This geometry is particularly relevant for thin-film materials, which tend to have crystalline domains with one crystallographic direction perpendicular to the substrate, but little or no orientation of the crystallites in the plane of the substrate. This symmetry also applies to fibres and to single-molecular-layer Langmuir films (Als-Nielsen et al., 1994; Kaganer et al., 1999; Kuzmenko et al., 2001). Liquid crystals and polymer films often exhibit broad in-plane orientation distributions. Anisotropy is a nuisance in the analysis of powder diffraction data, but for organic films with few Bragg reflections, it is a crucial source of structural information.

The specialized method of grazing-incidence X-ray diffraction (GIXD) has become well established for investigating the structure of films, surfaces and interfaces (Feidenhans'l, 1989; Dosch, 1992; Als-Nielsen \& McMorrow, 2001). The salient feature of GIXD is to enable studies of thin films on substrates, despite the strongly penetrating nature of hard $\mathrm{X}$-rays (photon energy $>8 \mathrm{keV}$ ). X-rays can be assigned an index of refraction $n=1-\delta_{n}+\mathrm{i} \beta_{n}$, where $\delta_{n}$ is of the order of $10^{-6}$, and the imaginary term with $\beta_{n} \simeq 10^{-8}$ accounts for absorption. $\delta_{n}$ and $\beta_{n}$ can be calculated from the wavelength, material stoichiometry and density. When the incoming beam hits the sample surface at a grazing-incidence angle smaller than the critical angle for total reflection $\alpha_{\mathrm{c}}$ (typically near $0.2^{\circ}$ ), the beam is totally reflected and only the rapidly decaying evanescent beam penetrates in towards the sample 
Table 1

The hierarchy of coordinate systems.

\begin{tabular}{lll}
\hline & Real space & Reciprocal space \\
\hline Crystallite & $\left(x_{\mathrm{c}}, y_{\mathrm{c}}, z_{\mathrm{c}}\right)$ & - \\
Sample & $\left(x_{\mathrm{s}}, y_{\mathrm{s}}, z_{\mathrm{s}}\right)$ & $\left(Q_{x}, Q_{y}, Q_{z}\right)$ \\
Laboratory & $\left(x_{\text {lab }}, y_{\text {lab }}, z_{\text {lab }}\right)$ & $\left(Q_{x, \text { lab }}, Q_{y, \text { lab }}, Q_{z, \text { lab }}\right)$ \\
\hline
\end{tabular}

interior. The signal from the bulk is thus strongly suppressed, effectively enhancing the signal-to-noise ratio for scattering from the surface layers. The penetration depth $\Lambda$ into the sample is of the order of $100 \AA$ for a typical wavelength of $1.5 \AA$ and an angle of incidence $\alpha_{\mathrm{i}}$ near $\alpha_{\mathrm{c}}$ ( $c f$. Feidenhans'l, 1989). When thin organic films on Si (or glass) substrates are studied, there are two critical angles, the critical angle of the film being smaller than that of the substrate. It is then possible to choose an incidence angle $\alpha_{\mathrm{i}}$ for which thin films of, say, 1-300 $\mathrm{nm}$ are fully penetrated by the beam, and the effective scattering volume is limited by the film thickness. Note that, for reasons of symmetry, in addition the exit angle must be larger than $\alpha_{\mathrm{c}}$ (Dosch, 1992).

In this article we shall review the kinematical framework necessary for simulating grazing-incidence wide-angle X-ray scattering, mainly with applications to organic thin films in mind. Particular attention is given to the degree of preferred orientation and the special case of two-dimensional powder symmetry.

To ease the reading, we now briefly present the applied simulation algorithm, which is a 'forward' approach in the sense that we start at the incoming beam and 'track' the X-rays through the sample and towards the detector. Especially for weakly scattering samples giving few reflections, combined with two-dimensional (area) detector devices, this is intuitively a faster approach than to calculate 'backwards' from every pixel of the detector. The algorithm is a compromise between 'user friendliness' and computational efficiency for simulating samples with varying degrees of cylindrical symmetry. Polycrystalline samples are modelled by adding the scattering contributions from a single crystal that sequentially assumes all the orientations of the crystalline domains of the actual sample. The scattering from disparate crystallites is assumed to be incoherent, and thus intensities rather than amplitudes are added.

The algorithm is as follows.

(1) For a specified crystallite orientation and lattice plane $(h k l)$, the reciprocal-lattice vector $\mathbf{G}_{h k l}$ is calculated. Note that $\mathbf{G}_{h k l}$ is generally different from the scattering vector $\mathbf{Q}$.

(2) Calculate whether the Laue diffraction condition $\mathbf{Q}=$ $\mathbf{G}_{h k l}$ can be fulfilled by rotating $\mathbf{G}_{h k l}$ (or, equivalently, the crystallite) about the sample plane normal. If it can, the corresponding scattering sample orientation and the resulting diffraction peak position are obtained.

(3) The peak intensity is calculated, accounting for geometrical correction factors, form and structure factors, and sample volume. If there is preferred orientation, the intensity is modified by a, typically Gaussian, function of the difference between the actual and the scattering crystallite orientations.
The organization of this article follows these points closely, with the subsequent three sections dedicated one-to-one to the steps of the algorithm. A two-beam model which accounts also for the totally substrate-reflected beam is described. Finally, the formalism is applied to a selected example from organic electronics.

The presented framework has been implemented in the computer program SimDiffraction with a graphical user interface, which can be obtained by contacting the first author. This article provides the basis for understanding and efficiently using the program. To the best of our knowledge, similar packages for simulating GIXD of polycrystalline films do not yet exist.

\section{Scattering geometry}

The conventional ' $2 \mathrm{~S}+2 \mathrm{D}$ ' geometry with a monochromatic beam is employed here, as it is frequently used for GIXD applied to thin films (Renaud et al., 1995; Vlieg, 1997; EvansLutterodt \& Tang, 1995; Kuzmenko et al., 2001; Bunk \& Nielsen, 2004). This geometry has two angles defining the sample orientation ( $\varphi$ about the sample normal, and the incidence angle $\alpha_{\mathrm{i}}$ ), and two detector angles, $\gamma$ (in-plane) and $\delta$ (out-of-plane). The scattering angle $2 \theta$ is determined by the two detector angles $\gamma$ (vertical) and $\delta$ (horizontal), as illustrated in Fig. 1, with $\cos 2 \theta=\cos \gamma \cos \delta$. The detector is at a distance $L$, 'the camera length', from the sample rotation axes.

In addition to the angles and dimensions of the instrument, the orientation of the individual crystallites must be parameterized. To this end, several right-handed orthogonal coordinate systems sharing the same origin are employed ( $c f$. Table 1), related to each other by standard rotation matrices; for example, for rotation by an angle $\eta$ about the $x$ axis,

$$
\mathbf{R}_{x}(\eta)=\left(\begin{array}{ccc}
1 & 0 & 0 \\
0 & \cos \eta & \sin \eta \\
0 & -\sin \eta & \cos \eta
\end{array}\right) .
$$

To parameterize the experimental setup, we use a laboratory coordinate system ( $\left.x_{\text {lab }}, y_{\text {lab }}, z_{\text {lab }}\right), y_{\text {lab }}$ being in the (horizontal) beam direction and $z_{\text {lab }}$ in the vertical. An associated reciprocal space coordinate system $\left(Q_{x, \text { lab }}, Q_{y, \text { lab }}, Q_{z \text {,lab }}\right)$, being collinear with $\left(x_{\text {lab }}, y_{\text {lab }}, z_{\text {lab }}\right)$ but having units of $\AA^{-1}$, is used in the later considerations.

It is expedient to consider also a sample coordinate system $\left(x_{\mathrm{s}}, y_{\mathrm{s}}, z_{\mathrm{s}}\right)$, with an associated reciprocal lattice $\left(Q_{x}, Q_{y}, Q_{z}\right)$, which is related to the laboratory system by two rotations $\mathbf{R}_{x}\left(\alpha_{\mathrm{i}}\right) \cdot \mathbf{R}_{z}(\varphi)$. The first rotation, by $\varphi$, is the sample rotation angle about the film normal; the second accounts for the incidence angle $\alpha_{\mathrm{i}}$. In the sample coordinate system, the surface angles $\alpha_{\mathrm{i}}, \alpha_{\mathrm{f}}$ and $\psi$ are defined as in Fig. 1(c). Because of the substrate, a sample horizon is introduced in the GIXD geometry, i.e. measurements cannot be performed for $\alpha_{\mathrm{f}}<0$. Note also that because the incidence angle is assumed fixed, the only specular point that can be reached is with $\alpha_{\mathrm{i}}=\alpha_{\mathrm{f}}=\delta / 2$ (and $\psi=\gamma=0$ ). There is thus a region of $Q$ space that cannot be explored using GIXD, as illustrated in Fig. 2.

At the lowest level of the hierarchy, each crystallite is in principle assigned an orthogonal coordinate system $\left(x_{\mathrm{c}}, y_{\mathrm{c}}, z_{\mathrm{c}}\right)$, 
(a) Side view

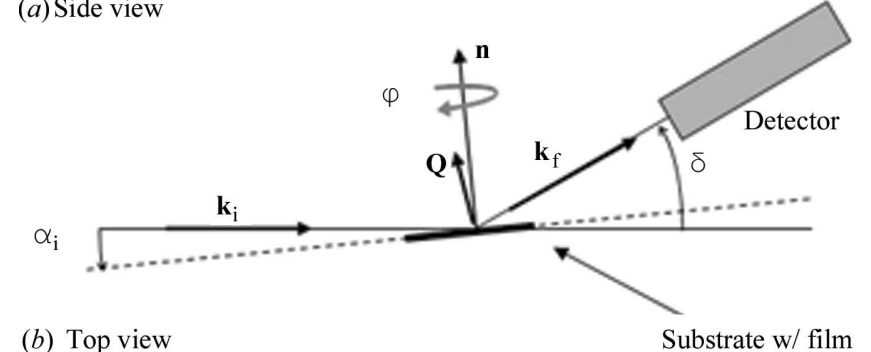

(b) Top view

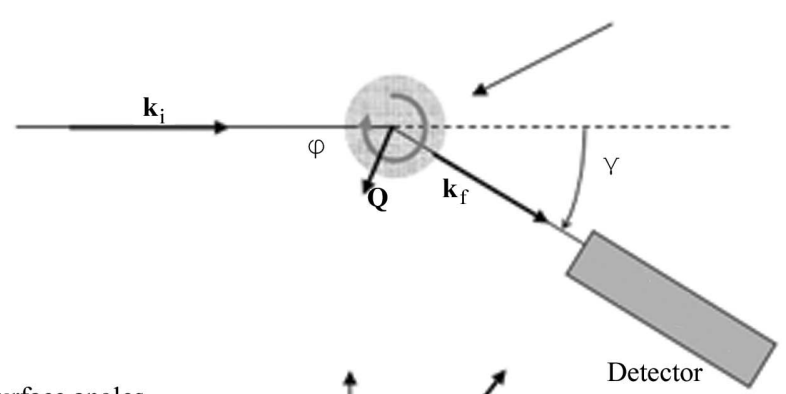

(c) Surface angles

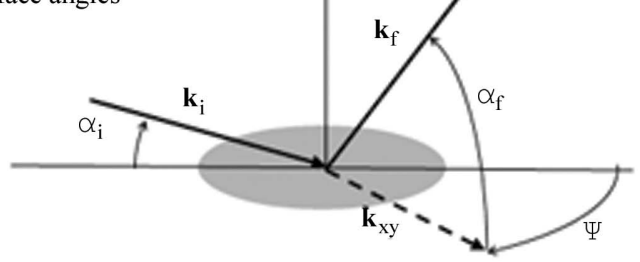

Figure 1

Sketch of the geometry of a grazing-incidence diffraction setup with a point detector, seen in $(a)$ side view and $(b)$ top view. Note that the drawings are not to scale. The rotation $\varphi$ is about the sample normal. The scattering vector $\mathbf{Q}$ is generally not parallel to the sample normal $\mathbf{n} .(c)$ Definition of the 'surface angles', $\alpha_{\mathrm{i}}$ being the incidence angle, $\alpha_{\mathrm{f}}$ the exit angle and $\psi$ the in-plane angle.

employing the 'Protein Data Bank (PDB) convention', which defines a along $\mathbf{x}_{\mathrm{c}}$, and the $a b$ plane to contain $\mathbf{y}_{\mathrm{c}}$. The reciprocal vector $\mathbf{c}^{*}$ will then be parallel to $\mathbf{z}_{\mathrm{c}}$. Thus, knowing the unit-cell parameters $a, b, c, \alpha, \beta$ and $\gamma$, the unit-cell vectors are obtained by $\mathbf{a}=(a, 0,0), \mathbf{b}=(b \cos \gamma, b \sin \gamma, 0)$ and $\mathbf{c}$ by $c_{x}=$ $c \cos \beta, c_{y}=\left(c \cos \alpha-c_{x} \cos \gamma\right) / \sin \gamma, c_{z}=\left(c^{2}-c_{x}^{2}-c_{y}^{2}\right)^{1 / 2}$. The reciprocal-lattice vectors $\mathbf{a}^{*}, \mathbf{b}^{*}, \mathbf{c}^{*}$ are calculated using the well known standard formulae. Any point in the reciprocal lattice associated with the crystallite can now be indexed by $\mathbf{G}_{0}$ $=h \mathbf{a}^{*}+k \mathbf{b}^{*}+l \mathbf{c}^{*}$, where $h, k, l$ are Miller indices (Als-Nielsen \& McMorrow, 2001).

In order to relate the $i$ th crystallite coordinate system to the sample coordinate system, we use the product of two operations, $\mathbf{W}^{i} \cdot \mathbf{R}$. The matrix $\mathbf{R}$ defines the easy axis, which can be considered the average of all crystallite orientations. $\mathbf{R}$ is taken to be the product of three successive rotations by angles $\rho_{x}, \rho_{y}$ and $\rho_{z}$, thus fully specifying the crystallite orientation in three dimensions. The first rotation by an angle $\rho_{y}$ is about the $y_{\mathrm{s}}$ axis, followed by $\rho_{x}$ about the $x_{\mathrm{s}}$ axis and finally $\rho_{z}$ about the $z_{\mathrm{s}}$ axis. A single $3 \times 3$ orientation matrix is obtained by taking $\mathbf{R}\left(\rho_{x}, \rho_{y}, \rho_{z}\right) \equiv \mathbf{R}_{z}\left(\rho_{z}\right) \cdot \mathbf{R}_{x}\left(\rho_{x}\right) \cdot \mathbf{R}_{y}\left(\rho_{y}\right)$. As $\mathbf{R}$ defines the dominant (average) orientation, coined the 'Frank director' in the special case of liquid crystals, it is natural to define $\mathbf{G}_{\mathrm{D}} \equiv$ $\mathbf{R G}_{0}$.

$\mathbf{W}^{i}$ denotes orientational deviations of the $i$ th crystallite from the predominant orientation, as will be described in more detail in $\$ 4.1$. For modelling polycrystalline systems with a distribution of orientations, we employ a set of values for the orientational deviations, $\left\{\mathbf{W}^{i}\right\}$. Intuitively, for a single crystal there is only one domain, and thus $\{\mathbf{W}\}=\mathbf{1}$. In summary, the reflection having indices $h k l$ is modelled by a set of vectors $\left\{\mathbf{G}_{h k l}\right\}$ in the sample coordinate system by

$$
\left\{\mathbf{G}_{h k l}\right\}=\left\{\mathbf{W}^{i}\right\} \mathbf{R}\left(\rho_{z}, \rho_{y}, \rho_{x}\right) \mathbf{G}_{0}(h, k, l)=\left\{\mathbf{W}^{i}\right\} \mathbf{G}_{\mathrm{D}} .
$$

For simplicity of notation, the remainder of this article refers to a single vector $\mathbf{G}_{h k l}$, which can be understood as any of the vectors contained in the set in (2). (a) $\alpha_{\mathrm{i}}=0^{\circ}$

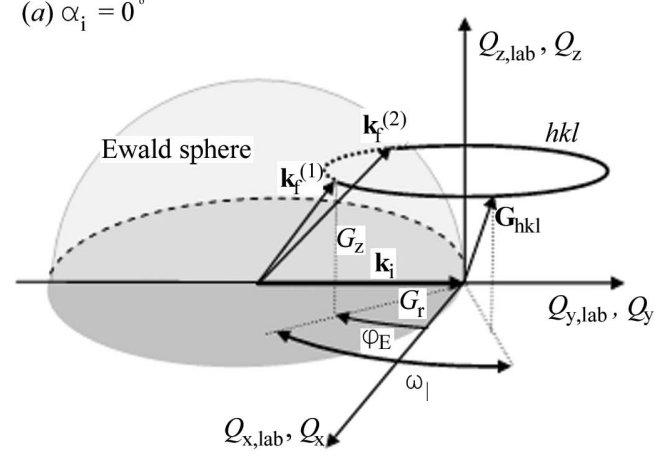

Figure 2

The diffraction condition for samples having cylindrical symmetry. $\mathbf{G}_{h k l}$ is the reciprocal-lattice vector to the plane $(h k l)$ of a given oriented crystallite. Rotating this vector $\mathbf{G}_{h k l}$ about the sample normal defines a 'ring' in reciprocal space, with radius $G_{\mathrm{r}}$ and height $G_{z}$. If this 'ring' intersects the Ewald sphere, the diffraction condition is fulfilled at two angles, $\varphi_{\mathrm{E}}^{(1)}$ and $\varphi_{\mathrm{E}}^{(2)}=180^{\circ}-\varphi_{\mathrm{E}}^{(1)}$, taken as the angles between the $Q_{x, \text { lab }}$ axis and the intersection points between the 'ring' and the Ewald sphere. At these intersection points, $\left|\mathbf{k}_{\mathrm{f}}\right|=\left|\mathbf{k}_{\mathrm{i}}\right|$, and $\mathbf{k}_{\mathrm{f}}=\mathbf{k}_{\mathrm{i}}+\mathbf{G}$. The angle $\omega_{\|}$is used to model samples with in-plane anisotropy, as it gives a measure of the orientational difference between the actual crystallite orientation and the diffraction condition. (a) Perspective drawing for $\alpha_{\mathrm{i}}=0$. (b) View along $Q_{x, \text { lab }}$ for $\alpha_{\mathrm{i}} \neq 0$.

\section{Diffraction from oriented powders}

The scattering vector is defined by $\mathbf{Q} \equiv$ $\mathbf{k}_{\mathrm{f}}-\mathbf{k}_{\mathrm{i}}$, where $\mathbf{k}_{\mathrm{i}}$ and $\mathbf{k}_{\mathrm{f}}$ denote the inand outgoing wavevectors, respectively. In the laboratory coordinate system, $\mathbf{k}_{\mathrm{i}}$ $=k \hat{\mathbf{y}}_{\text {lab }}$. For elastic scattering, $k \equiv\left|\mathbf{k}_{\mathrm{i}}\right|=$ $\left|\mathbf{k}_{\mathrm{f}}\right|=2 \pi / \lambda$, and $Q=|\mathbf{Q}|=2 k \sin \theta$. The Laue conditions for diffraction from lattice plane $(h k l)$ state that $\mathbf{Q}$ should be equal to the reciprocal-lattice vector $\mathbf{G}_{h k l}$. This condition is elegantly visualized by the Ewald construction in reciprocal space. When a reciprocallattice point intersects the Ewald sphere, diffraction takes place (Warren, 1969). The cylindrical symmetry of films with random inplane orientation of crystallites implies no $\varphi$ dependence; the lattice point $\mathbf{G}_{h k l}$ 
in reciprocal space is transformed into a 'ring' of radius $G_{\mathrm{r}}$ and 'height' $G_{z}$. For samples with a tendency of in-plane orientation, the density along this ring is a function of $\varphi$. Even in the case of full fibre symmetry it is crucial to know the orientation of the unit cell in terms of $\mathbf{W}^{i} \cdot \mathbf{R}$, as these parameters control the radius $G_{\mathrm{r}}$ and height $G_{z}$ of the ring. We transform $\mathbf{G}_{h k l}$ to cylindrical coordinates $\left(G_{\mathrm{r}}, \varphi, G_{z}\right)$ by taking

$$
\begin{aligned}
G_{\mathrm{r}} & =\left(G_{x}^{2}+G_{y}^{2}\right)^{1 / 2}, \quad G_{z}=G_{z}, \quad G=\left(G_{\mathrm{r}}^{2}+G_{z}^{2}\right)^{1 / 2}, \\
G_{x} & =G_{\mathrm{r}} \cos \varphi, \quad G_{y}=-G_{\mathrm{r}} \sin \varphi .
\end{aligned}
$$

Here, $G \cong\left|\mathbf{G}_{h k l}\right|$, and the angle $\varphi$ in the $x y$ plane is defined to be zero on the $Q_{x, \text { lab }}$ axis and positive clockwise.

The diffraction condition for a system with cylindrical symmetry is that the above-mentioned $\mathbf{G}_{h k l}$ 'ring' intersects the Ewald sphere, thus fulfilling the Laue condition for a given $(h k l)$ lattice plane. Dropping the $h k l$ subscript for clarity, we let $\mathbf{G}_{\mathrm{E}}$ denote a vector that fulfils the diffraction condition. It is clear from Fig. 2 that, by symmetry, there are two intersection points between the ring and the Ewald sphere. We let $\varphi_{\mathrm{E}}^{(1)}$ and $\varphi_{\mathrm{E}}^{(2)}$ denote the angles between the $Q_{x, \mathrm{lab}}$ axis and these intersection points. The second solution $\varphi_{\mathrm{E}}^{(2)}=180^{\circ}-\varphi_{\mathrm{E}}^{(1)}$ corresponds to $G_{\mathrm{E}, x} \rightarrow-G_{\mathrm{E}, x}$. Clearly, in the special case that the $h k l$ ring barely touches the Ewald sphere, there will be just one solution at $\varphi_{\mathrm{E}}=90^{\circ}$. Our way to proceed is thus to calculate the angle $\varphi_{\mathrm{E}}$ of the intersection point (cf. Fig. 2). To this end, the Ewald sphere is parameterized by

$$
Q_{x, \text { lab }}^{2}+\left(Q_{y, \text { lab }}+k\right)^{2}+Q_{z, \text { lab }}^{2}=k^{2} .
$$

By applying a rotational transformation $\mathbf{R}_{x}\left(\alpha_{\mathrm{i}}\right)$ as in (1) and (2), one obtains $\mathbf{G}_{h k l}=\left\langle G_{\mathrm{r}} \cos \varphi,-G_{\mathrm{r}} \sin \varphi \cos \alpha_{\mathrm{i}}-G_{z} \sin \alpha_{\mathrm{i}}\right.$, $\left.-G_{\mathrm{r}} \sin \varphi \sin \alpha_{\mathrm{i}}+G_{z} \cos \alpha_{\mathrm{i}}\right\rangle$. By inserting these components into (4), it is straightforward to show that

$$
\sin \varphi_{\mathrm{E}}=\frac{1}{2 k} \frac{G^{2}}{G_{\mathrm{r}}} \frac{1}{\cos \alpha_{\mathrm{i}}}-\frac{G_{z}}{G_{\mathrm{r}}} \tan \alpha_{\mathrm{i}} .
$$

This equation yields solutions for $\varphi_{\mathrm{E}}^{(1)}$ in the interval $\left(0,90^{\circ}\right)$, as it should. Having obtained $\varphi_{\mathrm{E}}$, the components $G_{\mathrm{E}, x}, G_{\mathrm{E}, y}$ and $G_{\mathrm{E}, z}$ are readily calculated from (3). For an in-plane reflection $\left(G_{\mathrm{E}, z} \simeq 0, G \simeq G_{\mathrm{r}}\right)$ and a grazing-incidence angle $\alpha_{\mathrm{i}}$ $\simeq 0, G_{\mathrm{E}, y}$ has a particularly simple solution; $G_{\mathrm{E}, y}=-G^{2} / 2 k$, and the standard expression $Q=2 k \sin \varphi_{\mathrm{E}}$ is retrieved when noting that $Q=G$ and that $\varphi_{\mathrm{E}}$ is then the Bragg angle.

\section{Intensity calculations}

Several factors determine the observable intensity $I_{h k l}$ of a Bragg reflection in GIXD geometry; it can be expressed by a slight modification of the general expression found, for example, in the book by Als-Nielsen \& McMorrow (2001):

$$
\begin{aligned}
I_{h k l}(\mathbf{Q})= & \sum_{\left\{W^{i}\right\}}\left[\Phi_{0} r_{0}^{2} N_{\mathrm{c}}\left(\lambda^{3} / v_{\mathrm{c}}\right) \Omega P(\gamma, \delta) L(\gamma, \delta)\right. \\
& \left.\times V_{\mathrm{eff}}(\gamma, \delta)|F(\mathbf{Q})|^{2}\right] .
\end{aligned}
$$

Here, $\Phi_{0}$ denotes the incoming flux, $r_{0}=2.82 \times 10^{-15} \mathrm{~m}$ is the classical electron radius and the sum is over all orientations $\left\{\mathbf{W}^{i}\right\} ; N_{\mathrm{c}}$ denotes the total number of unit cells and $v_{\mathrm{c}}$ the unit- cell volume. The Lorentz $L(\gamma, \delta)$ and polarization $P(\gamma, \delta)$ factors have been treated in detail by Smilgies (2002) and will not be repeated here. Some comments on the effective volume $V_{\text {eff }}$ are given below, and separate sections are devoted to the corrections for preferred orientation $\Omega$ and also to refraction.

The structure factor $F(\mathbf{Q})$, the key quantity for the crystallographer, can be calculated from the atomic form factors (also called scattering factors) $f_{j}$ (Wilson, 1995) and the relative atomic positions $\mathbf{r}_{j}=\left(x_{j}, y_{j}, z_{j}\right)$, as described in standard textbooks:

$$
F(\mathbf{Q})=\sum_{j} f_{j}(Q) \exp \left[2 \pi i\left(h x_{j}+k y_{j}+l z_{j}\right)\right] \exp \left(-M_{j}\right) .
$$

$M_{j}$ is the Debye-Waller factor. The atomic form factors $f(Q)$ for the elements can be found tabulated on the Web (e.g. $\mathrm{http} / /$ www.cxro.lbl.gov) or in references such as International Tables for X-ray Crystallography (Wilson, 1995). Except near absorption edges, a Cromer-Mann expansion describes the atomic form factor as a function of energy quite well (Cromer \& Mann, 1968), and this approach is implemented in the software described here, with parameters as listed by Wilson (1995).

The (lateral) geometrical variation of $V_{\text {eff }}$ as a function of the detector angles $(\gamma, \delta)$ is treated for a collimated point detector by Smilgies (2002). When using a two-dimensional detector, however, the scattering from the whole beam footprint reaches the detector, and $V_{\text {eff }}$ is thus a constant. Because the effective scattering volume $V_{\text {eff }}$ of thin films is highly restricted ('truncated') in the direction perpendicular to the film substrate, the resulting diffraction image is modified by the fact that a limited number of unit-cell repetitions are available. The truncation of the crystallites leads to a continuous intensity profile connecting Bragg points in the $Q_{z}$ direction, i.e. perpendicular to the surface plane. These socalled crystal truncation rods (CTRs) (Robinson, 1986; Feidenhans'l, 1989; Als-Nielsen \& McMorrow, 2001; Smilgies et al., 2005) can also be observed for well ordered organic thin films (Gidalevitz et al., 1997; Breiby et al., 2005). The modification of the scattering pattern by CTRs can be considered as introducing a convolution by the Fourier transform of the (thin film) sample geometry.

\subsection{Preferred orientation}

$\Omega$ in (6) is a correction for preferred orientation, which is vital for this study. The total scattering from all crystallite orientations is calculated as the incoherent sum of scattering from all orientations $\left\{\mathbf{W}^{i}\right\}$ in (2), appropriately weighted to account for preferred orientation as described by an orientational distribution function (ODF). As this is in fact a way of estimating an integral over all orientations, it is clear that the form of $\left\{\mathbf{W}^{i}\right\}$ should be chosen in an efficient way for sampling the ODF, while keeping the computational burden reasonably low. A compromise must thus be found between angular step lengths and numerical accuracy. In principle, for an isotropic powder sample, $\left\{\mathbf{W}^{i}\right\}$ should cover all orientations, and the weighting should be uniform. Of course, because there are much more efficient ways of simulating a powder, we will not 
discuss further this special case. For samples with a pronounced degree of preferred orientation, there is no need to spend time performing calculations for orientations contributing negligibly to the scattered intensity. We remind the reader that the 'main' (or average) crystallite orientation is described by $\mathbf{W}=\mathbf{1}$.

For fully specifying the orientation of a rigid body, three rotations are needed. Still, it is often seen that only two rotations are employed, since for uniaxial samples, the orientation about the unique axis of the crystallites (the axis that exhibits preferred orientation) is implicitly assumed to be isotropic. Empirically, in thin-film samples with preferred orientation, the crystallites tend to orient with one crystal plane parallel to the substrate, and if special treatments have been applied to the substrate, there may also be preferred inplane orientation. Thus, it is often convenient to consider orientation angles $\omega_{\perp}$ and $\omega_{\|}$, describing the angular deviations of the crystallites from the average orientation with respect to (i) the sample normal and (ii) an in-plane axis of preferred orientation, respectively. Azimuth offset angles $\omega_{\|}$, describing the (azimuthal) crystallite orientations about the sample plane normal ('in-plane'), are obtained from

$$
\omega_{\|}^{(1),(2)}=\varphi_{\mathrm{E}}^{(1),(2)}-\varphi_{0} .
$$

For each crystallite orientation, there are two $\omega_{\|}$values, because of the two intersection points of the Ewald sphere ( $c f$. Fig. $2 a)$. The angle $\varphi_{0}$ is obtained by $\varphi_{0}=-\arctan \left(G_{h k l, y} /\right.$ $\left.G_{h k l, x}\right) \cdot \omega_{\|}$is thus the in-plane difference angle between the orientation that gives diffraction $\left(\varphi_{\mathrm{E}}\right)$ and the actual orientation $\left(\varphi_{0}\right)$ (see Fig. 2). In short, $\omega_{\|}$describes by which angle from its present position the sample must be rotated about the sample plane normal to fulfil the diffraction condition (if at all possible). For samples with full two-dimensional powder (cylinder) symmetry, the intensity is independent of $\omega_{\|}$. Similarly, for the out-of-plane anisotropy we use the angle $\omega_{\perp}$, which is the polar angle ('out-of-plane') difference between the actual orientation and the easy axis, obtained by

$$
\omega_{\perp}=\chi_{\mathrm{E}}-\chi_{0} .
$$

The angle $\chi_{0} \cong \arccos \left(G_{\mathrm{D}, z} / G_{\mathrm{D}}\right)$ is a polar angle in reciprocal space, obtained for the average orientation $\mathbf{G}_{\mathrm{D}}(c f . \S 2)$. Accordingly, $\chi_{\mathrm{E}} \cong \arccos \left(G_{\mathrm{E}, z} / G_{\mathrm{E}}\right)$ is the polar angle corresponding to the actual intersection with the Ewald sphere. For samples not exhibiting out-of-plane orientation, all values of $\omega_{\perp}$ are assigned equal weight. Note that the use of only two angles, the polar $\omega_{\perp}$ and the azimuthal $\omega_{\|}$, is sufficient to describe a wide range of different ODFs, but it still poses a limitation on the ODFs that can be described. For example, this geometry does not cover the case of cylindrical symmetry with the cylinder axis being in-plane, which is relevant, for example, for certain liquid crystals with the Frank director parallel to the substrate. However, in the present context, this omission is made to simplify the description of the geometry.

4.1.1. Biaxial model. As the simplest conceivable model for biaxial samples, we suggest the product of two Gaussian functions,

$$
\Omega\left(W_{\|}, W_{\perp}\right)=\frac{1}{N} \exp \left(\frac{-\omega_{\|}^{2}}{W_{\|}^{2}}\right) \exp \left(\frac{-\omega_{\perp}^{2}}{W_{\perp}^{2}}\right) .
$$

The parameters $W_{\|}$and $W_{\perp}$ control the in-plane and out-ofplane degree of orientation, respectively, and $N$ is a normalization factor. Note that because we prefer to distinguish explicitly between $h k l$ and $\bar{h} \bar{k} \bar{l}$, this distribution function does not have the $180^{\circ}$ periodicity sometimes seen, for example, in the Maier-Saupe distribution (Breiby \& Samuelsen, 2003). In the case of isotropic samples, $W_{\|}$and $W_{\perp} \rightarrow \infty$, giving $\Omega=1$. If approaching a single crystal, $W_{\|}$and $W_{\perp} \rightarrow 0$. Cylinder (uniaxial) symmetry about a preferred vertical axis is obtained by $W_{\|} \rightarrow \infty$. For samples with moderate biaxial orientation, $W_{\|} \simeq W_{\perp} \simeq 5-30^{\circ}$. Clearly, equation (10) can be replaced by other ODFs depending on the problem in hand, or it can be combined with, for example, mirroring about symmetry axes.

The associated crystallite orientations $\left\{\mathbf{W}^{i}\right\}$ of equation (2) are calculated from a 'distribution angle' $\omega_{\perp}^{i}$, which denotes a rotation of the $i$ th crystallite that keeps $\mathbf{G}_{\mathrm{D}}$ on its current meridian (thus only changing the polar angle). For the simulations, $\left\{\omega_{\perp}\right\}$ is provided by the user, and for a choice of, for example, $\left\{\omega_{\perp}\right\}=\left\{-5^{\circ},-4^{\circ}, \ldots, 5^{\circ}\right\}$, the orientation distribution function (ODF) is probed at 11 points around the dominant orientation $\mathbf{G}_{\mathrm{D}}$. Clearly, the range of $\left\{\omega_{\perp}\right\}$ should be chosen to be sufficiently wide to cover the tails of the ODF, and the spacing should be sufficiently close to obtain a reliable estimate of the intensity.

4.1.2. Kratky model. Since it is a common description for uniaxial orientation, we continue with a brief presentation of our implementation of the classical Kratky (1933) model, which describes the alignment of rigid rods (crystallites) in an affine matrix stretched by an elongation ratio $\lambda_{\mathrm{K}}$. According to this model, the unique axis of a crystallite (in polymers usually the chain axis) orients at an angle $\omega_{\perp}$ with respect to the dominant orientation $\mathbf{G}_{\mathrm{D}}$ according to an $\operatorname{ODF} f\left(\omega_{\perp}\right)$, given by

$$
f\left(\omega_{\perp}\right)=\frac{1}{4 \pi} \frac{\lambda_{\mathrm{K}}^{3}}{\left[\lambda_{\mathrm{K}}^{3}-\left(\lambda_{\mathrm{K}}^{3}-1\right) \cos ^{2} \omega_{\perp}\right]^{3 / 2}} .
$$

On average, the crystallites orienting with a certain $\omega_{\perp}$ assume all possible rotation angles about the unique axis, all of which contribute equally to the scattered intensity. Thus, in the Kratky model, the following parameters are taken as input to the simulation algorithm: the elongation ratio $\lambda_{\mathrm{K}}$ governing the distribution width, the set of angles $\left\{\omega_{\perp}\right\}$ and an angular step length for the full rotation of each crystallite about its unique axis.

In the important limit of a high degree of cylindrical symmetry (biaxial model: $W_{\perp} \rightarrow 0, W_{\|} \rightarrow \infty$; Kratky model: $\left.\lambda_{\mathrm{K}} \rightarrow \infty\right)$, the two presented models give the same result.

\subsection{Refraction}

Refraction effects are governed by Snell's law, $n_{0} \cos \xi_{0}=$ $n_{1} \cos \xi_{1}$, where subscripts 0 and 1 denote different materials and the angles $\xi_{0}$ and $\xi_{1}$ are defined with respect to the interface (rather than the interface normal). Because $n$ decreases with increasing electron density, total reflection 
occurs below a (small) critical angle $\alpha_{c}=2^{1 / 2}\left(1-n_{1} / n_{0}\right)^{1 / 2}$ when for instance going from a vacuum towards a solid surface (Als-Nielsen \& McMorrow, 2001). In the common case of $n_{0}=$ 1 (vacuum), the expression for $\alpha_{\mathrm{c}}$ simplifies to $\left(2 \delta_{1}\right)^{1 / 2}$. Albeit frequently neglected, the effects of refraction on the peak positions and intensities can be substantial, especially when dealing with grazing-incidence small-angle X-ray scattering (GISAXS). GISAXS data are sometimes modelled in a "quasikinematical' (Naudon, 1995) approach by a product of three factors: the molecular form factor $F_{\mathrm{mol}}(Q)$ (which gives features at lower $Q$ than is usually observed in wide-angle $\mathrm{X}$-ray scattering), the structure factor $F(Q)$ and the Vineyard (1982) factor $T\left(Q_{z}\right)$ observed for small exit angles near $\alpha_{\mathrm{c}}$. Much more advanced GISAXS descriptions have since been developed (Sinha et al., 1988; Busch et al., 2006), resulting in the well known IsGISAXS computer program of Lazzari (2002).

A notable effect of refraction is to reduce the incidence angle $\alpha_{\mathrm{i}}$ to an effective incidence angle $\alpha_{\mathrm{i} \text {,eff }}$ (cf. Fig. 3). Except for in-plane reflections, the outgoing beam generally has an exit angle $\alpha_{\mathrm{f}} \gg \alpha_{\mathrm{c}}$, and the refraction of this beam is therefore less pronounced. Experimentally, the most obvious effect of refraction is thus a shift of the peaks towards higher $Q_{z}$ (Toney \& Brennan, 1989).

Another effect pertinent to GIXD is that for $\alpha_{\mathrm{i} \text {, ff }}<\alpha_{\mathrm{c}}$, the beam reflected totally off the substrate will contribute to the total scattering also when traversing the film on its way 'up' towards the film-air interface. (For $\alpha_{\mathrm{i}, \text { eff }}>\alpha_{\mathrm{c}}$, a weaker beam, as governed by the Fresnel equations, will be reflected.) As a result, for samples with well resolved Bragg peaks, a peak splitting can often be observed with an angular separation corresponding to essentially $2 \alpha_{\mathrm{i}, \text { eff }}(c f$. Fig. 3 ). This 'secondary' beam can be included in the simulations by adding the scattering pattern arising from calculations with an incidence angle of $-\alpha_{\mathrm{i}, \text { eff }}$. As can be seen from (5), changing $\alpha_{\mathrm{i}, \text { fff }} \rightarrow$ $-\alpha_{\mathrm{i}, \text { fff }}$ gives a negligible change of $\varphi_{\mathrm{E}}$, and thus of $\mathbf{G}_{\mathrm{E}}$ and $\mathbf{k}_{\mathrm{f}}$, for small $\alpha_{\mathrm{i} \text {,ff }}$. Because the formalism presented in this article

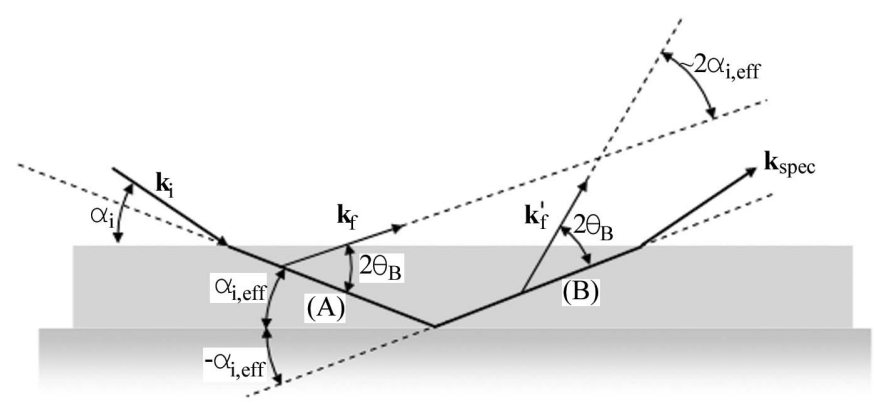

\section{Figure 3}

The influence of total substrate reflection and refraction on the scattering from a thin film on a substrate. The incoming beam $\mathbf{k}_{\mathrm{i}}$ with incidence angle $\alpha_{\mathrm{i}}$ is refracted to give an effective incidence angle $\alpha_{\mathrm{i}, \text { eff }}$. When $\alpha_{\mathrm{i} \text {,eff }}<$ $\alpha_{\mathrm{c} \text {,substrate }}$ the incoming beam will be totally reflected; $\mathbf{k}_{\mathrm{spec}}$ denotes the specularly reflected beam. The resulting scattering is the superposition of scattering from the 'primary' beam (A) going into the film and from the totally substrate-reflected 'secondary' beam (B). Inside the film, the

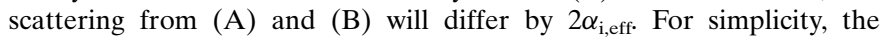
refraction of the beams $\mathbf{k}_{\mathrm{f}}$ and $\mathbf{k}_{\mathrm{f}}^{\prime}$ is suppressed in this figure. Note that the angles $\alpha_{\mathrm{i}}$ and $\alpha_{\mathrm{i} \text {,eff }}$ are greatly exaggerated for clarity. assumes that $\mathbf{k}_{\text {in }}$ is parallel to $y_{\text {lab }}, \mathbf{k}_{\mathrm{f}}^{\prime}$ is rotated by $2 \alpha_{\mathrm{i}, \text { eff }}$ before its associated detector angles $\gamma^{\prime}$ and $\delta^{\prime}$ are calculated, as described later. Even though the two-beam approach described here is a simplification compared with the four contributions often considered in GISAXS (Sinha et al., 1988), the validity of the two-beam approximation has been recently investigated by Lee et al. (2005) and Busch et al. (2006) and seems to hold in most cases. More rigorous models would also include effects such as surface roughness and standing waves, but the presented formalism gives good predictions for many purposes.

\section{Point-detector scans}

The preceding discussion has concerned simulating diffraction images, as recorded using a CCD detector or other twodimensional imaging device. For completeness, we add a few considerations about simulating scans performed with a point detector. In the SimDiffraction computer program, this is carried out in a slightly different way. The reciprocal-space lattice (containing nodes with structure factors $\left|F_{h k l}\right|^{2}$ ) is calculated from the (known) real-space crystal lattice with the predominant orientation $\mathbf{G}_{\mathrm{D}}$. The simulated diffraction scans are then obtained by calculating a trace through reciprocal space, where for every discrete point $j$, the intensity is obtained by the approximation

$$
\begin{aligned}
I_{j}= & \sum_{n} \mathrm{LPV}\left|F_{h k l}\right|^{2} P_{1}\left(W_{Q}, Q_{j}-Q_{n}\right) \\
& \times P_{2}\left(W_{\perp}, \chi_{j}-\chi_{n}\right) P_{3}\left(W_{\|}, \varphi_{j}-\varphi_{n}\right) .
\end{aligned}
$$

The sum is over all reciprocal-lattice points $n$. LPV denotes the Lorentz, polarization and volume corrections, all depending on the detector angles. Because the detector has a fixed position for in-plane rocking scans (constant $Q$ ), the LPV factors contribute only a multiplicative factor in this case. The weighting factors $P_{i}$ in equation (12) are peak functions of width $W_{i}$, the second argument being the offset from the peak maximum. The pseudo-Voigt $P_{1}$ measures the radial distance ( $Q$ mismatch, units $\AA^{-1}$ ) from the current point $j$ to lattice point $n$, and the width $W_{Q}$ arises from mosaic spread and instrumental resolution. Similarly, out-of-plane and in-plane preferred orientation are accounted for by pseudo-Voigt functions $P_{2}$ and $P_{3}$, respectively.

\section{Presenting the simulations}

Diffraction data are often presented with the scattered intensity as function of $Q, Q_{x}, Q_{y}$ or $Q_{z}$. For displaying the diffraction (Bragg) peaks for a two-dimensional powder, the most natural approach is to plot the reflections in the $Q_{x y} Q_{z}$ plane, with $Q_{x y} \equiv\left(Q_{x}{ }^{2}+Q_{y}{ }^{2}\right)^{1 / 2}$. From the out-going wavevector $\mathbf{k}_{\mathrm{f}}=\mathbf{G}_{\mathrm{E}}+\mathbf{k}_{\mathrm{in}}$ the detector angles $(\gamma, \delta)$ are easily calculated; by defining $\mathbf{k}_{x y}$ as the projection of $\mathbf{k}_{\mathrm{f}}$ onto the $x_{\text {lab }} y_{\text {lab }}$ plane (cf. Fig. 1) we have

$$
\gamma=\arccos \left(\hat{\mathbf{y}} \cdot \mathbf{k}_{x y} /\left|\mathbf{k}_{x y}\right|\right)
$$

and 


$$
\delta=\arccos \left(\mathbf{k}_{\mathrm{f}} \cdot \mathbf{k}_{x y} /\left|\mathbf{k}_{\mathrm{f}}\right|\left|\mathbf{k}_{x y}\right|\right) .
$$

Considering a two-dimensional detector, it is also straightforward to calculate the pixel coordinates $\left(p_{x}, p_{z}\right)$ with respect to the beam centre, as $p_{x}=\left(L / d_{\mathrm{p}}\right) \tan \gamma$ and $p_{z}=[(L / \cos \gamma) /$ $\left.d_{\mathrm{p}}\right] \tan \delta$, where $L$ is the camera length and $d_{\mathrm{p}}$ is the pixel size. Note that because the incoming beam is approximately parallel to the substrate, the resulting peak broadening caused by the extended footprint of the beam can be accounted for by allowing a range of values for $L$.

The experimental peak width is a convolution of the instrumental resolution and the intrinsic width relating to the sample. With synchrotron radiation, the instrumental resolution is sometimes negligible when compared with the broad peaks often observed for organic samples. Neglecting strain in the material, a simple parameterization of the peak width in terms of crystallite size $\xi$ is given by the Scherrer formula (see e.g. Warren, 1969). The peak width can be accounted for by convoluting the simulated diffractogram with an appropriate function. Typical choices are Gaussian or Lorentzian peak shapes, or combinations of these (Voigt or pseudo-Voigt) profiles. We note that for many setups, especially home laboratory instruments, the instrumental resolution function may prove detrimental to a meaningful analysis of the peak widths.

\section{Practical example: perylene}

As mentioned in $\S 1$, further progress in nanotechnology requires improved methods for inducing and characterizing material order. To illustrate the concepts treated in this article, we have chosen perylene as a relevant example from organic small-molecule electronics. Perylene is much studied both for fundamental chemistry reasons and as a potential building block in future molecular electronics. For simulating the diffraction data the unit cell advanced by Camerman $\&$ Trotter (1964) was used. This cell is monoclinic: $a=11.28, b=10.83, c=$ $10.26 \AA$ and $\beta=100.55^{\circ}$ ( $c f$. Fig. 4 for an illustration).

Small crystallites of height $\sim 70 \mathrm{~nm}$ and lateral size of up to $1 \mu \mathrm{m}$ ( $c f$. Breiby et al., 2008) were obtained by chemical vacuum deposition (CVD) perpendicularly onto (1) a clean silicon substrate and (2) a silicon substrate with frictiondeposited polytetrafluoroethylene (PTFE, 'Teflon') for inducing in-plane orientation (Wittman \& Smith, 1991). Si substrates for CVD were cleaned by boiling in photoresist remover, followed by ultrasonic cleaning in first acetone and then dichloromethane for $10 \mathrm{~min}$, before drying in filtered $\mathrm{N}_{2}$ gas flow. For the PTFE deposition, the substrates were wiped off with ethanol.

Using the rotating-anode-based GIXD setup at Ris $\varnothing$ National Laboratory, the images shown in Figs. 5(a) and 6(a) were captured with a Fuji image plate having a resolution ('pixel size') of $50 \times 50 \mu \mathrm{m}$. The wavelength $\lambda$ was $1.5418 \AA$ $(\mathrm{Cu} K \alpha)$, monochromated by a multilayer X-ray mirror. The incoming beam had dimensions of approximately $0.4 \times$ $0.4 \mathrm{~mm}$ and impinged onto the sample substrate at an incidence angle of $0.20(1)^{\circ}$ with a considerable divergence. This gives a footprint of $\sim 0.4 / \sin \left(0.2^{\circ}\right) \mathrm{mm}=115 \mathrm{~mm}$, which implies that the diameter of the sample limits the effective scattering volume. The thin film and the image plate at a distance of $L=120 \mathrm{~mm}$ from the sample were mounted in an evacuated chamber.

Separate synchrotron measurements were carried out at the $z$-axis diffractometer associated with the BW-2 beamline at HASYLAB in Germany, using a wavelength $\lambda=1.240 \AA$ and an incidence angle of $0.158^{\circ}$. A special adapter with a $\varphi$ rotation was employed, giving a vertical sample surface normal [see Bunk \& Nielsen (2004) for further details]. The last defining slit $\sim 150 \mathrm{~mm}$ upstream of the sample was adjusted to give a beam size of $1 \mathrm{~mm}$ (horizontal) by $0.01 \mathrm{~mm}$ (vertical). This gives a footprint of only $\sim 3.6 \mathrm{~mm}$, well within the sample diameter of about $20 \mathrm{~mm}$, thus ensuring that no intensity artefacts caused by a changing scattering volume arise during sample $\varphi$ rotations. The diffracted intensity was measured with a Cyberstar point detector.

As demonstrated in Figs. 5 and 6, excellent fits are obtained when assuming that the $a b$ plane of the unit cell is oriented parallel to the substrate (i.e. $\mathbf{R}=\mathbf{1}$ ). As it turned out, the degree of orientation for the sample cast on Si (no in-plane orientation) was sufficiently high that, within the experimental accuracy, the correction for preferred orientation $\Omega$ could be taken as unity in the simulations. In fact, tangentially, the peaks in Fig. 5( $a)$ can be well fitted by Gaussians of FWHM $\simeq$ $0.7-0.8^{\circ}$, which suggests that the crystallites are grown directly onto the substrate giving a 'perfect' (001) orientation, only smeared by the instrumental resolution. In the magnification

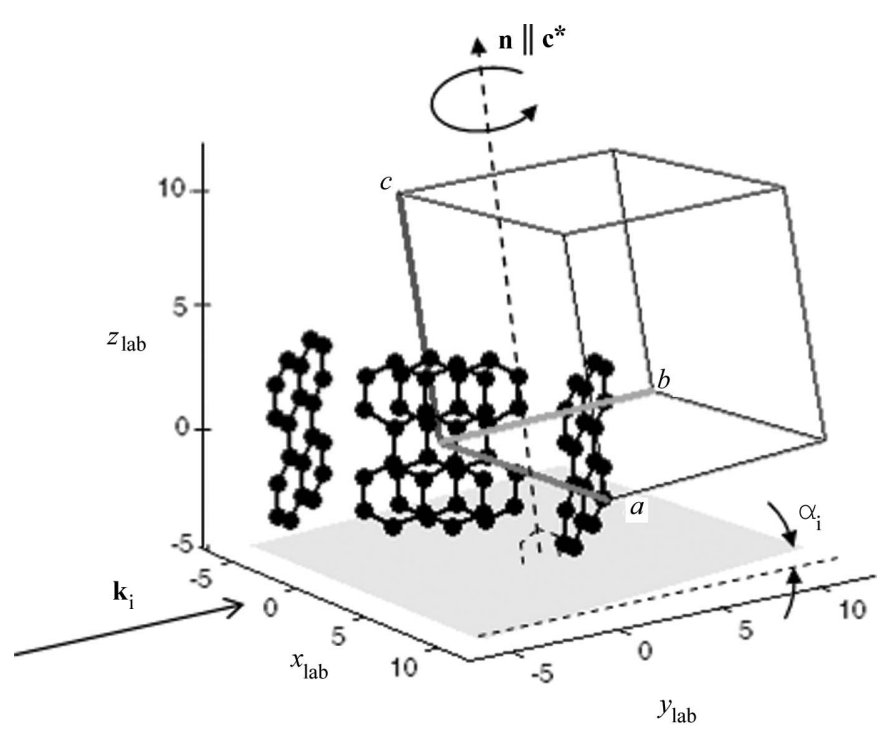

Figure 4

Schematic drawing of the monoclinic unit cell of perylene, having four molecules per unit cell, shown in the laboratory coordinate system with $\mathbf{k}_{\mathrm{i}}$ $\| \mathbf{y}_{\text {lab }}$. The unit cell is drawn with the $a b$ plane parallel to the substrate, which gives $\mathbf{c}^{*}$ parallel to the sample normal. The incidence angle $\alpha_{\mathrm{i}}$ is exaggerated for clarity. The $x_{\mathrm{s}} y_{\mathrm{s}}$ plane of the sample coordinate system, which is parallel to the sample substrate, is indicated by the shaded grey rectangle. If exhibiting cylindrical symmetry, the sample contains crystallites having all possible orientations in the plane of the substrate, as indicated by the circular arrow. 
of Fig. 5(c), the splitting caused by the two-beam effect is clearly seen and faithfully reproduced, using $\alpha_{\mathrm{i}}=0.20^{\circ}$ and an effective incidence angle $\alpha_{\mathrm{i}, \mathrm{eff}} \simeq 0.12^{\circ}$. For this sample, a $3 \mathrm{~mm}$ footprint correction was applied, which is the main contribution to the radial width of the peaks.

The image in Fig. 6(a) for perylene oriented on PTFE is very different from that obtained for the sample with cylindrical symmetry in Fig. 5. To obtain the former, the sample was
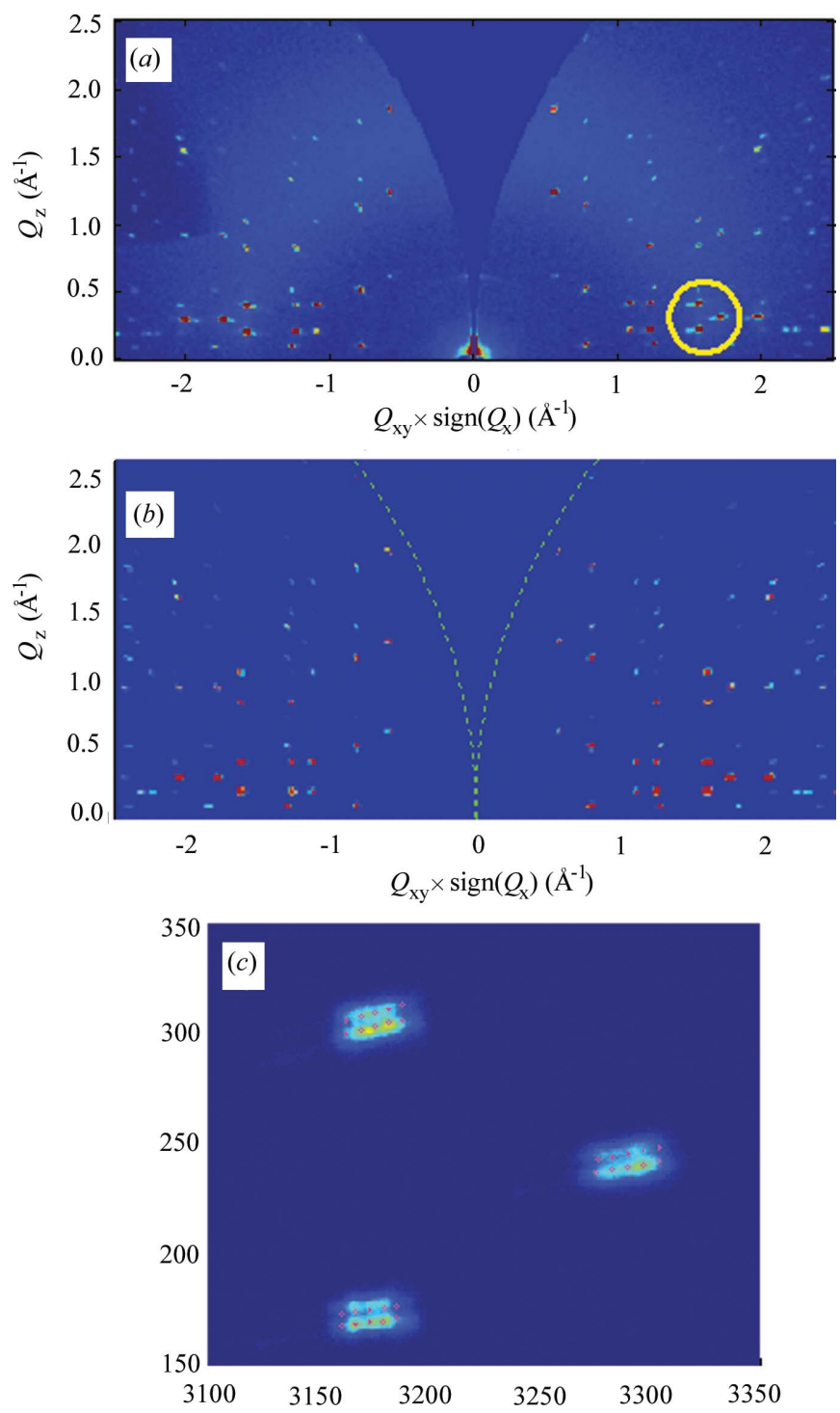

\section{Figure 5}

Comparison of $(a)$ the experimental diffraction image and $(b)$ the simulated image assuming a cylindrical distribution of perylene crystallites having the $a b$ plane parallel to the Si substrate. The agreement of the perylene peak positions, widths and intensities is very good. (c) Magnification of the original raw data (pixel coordinates) corresponding to the encircled region in $(a)$. The peak splitting caused by scattering from both the incident and the reflected beams is clearly seen. The magenta circles indicate the simulated peak positions, including refraction in the two-beam approximation. The radial peak broadening is accounted for discretely by calculating for different camera lengths $L$, varying from the nominal $120 \mathrm{~mm}$ by $\{-1.5,-0.75, \ldots, 1.5\} \mathrm{mm}$, thus corresponding to a beam footprint of $3 \mathrm{~mm}$.
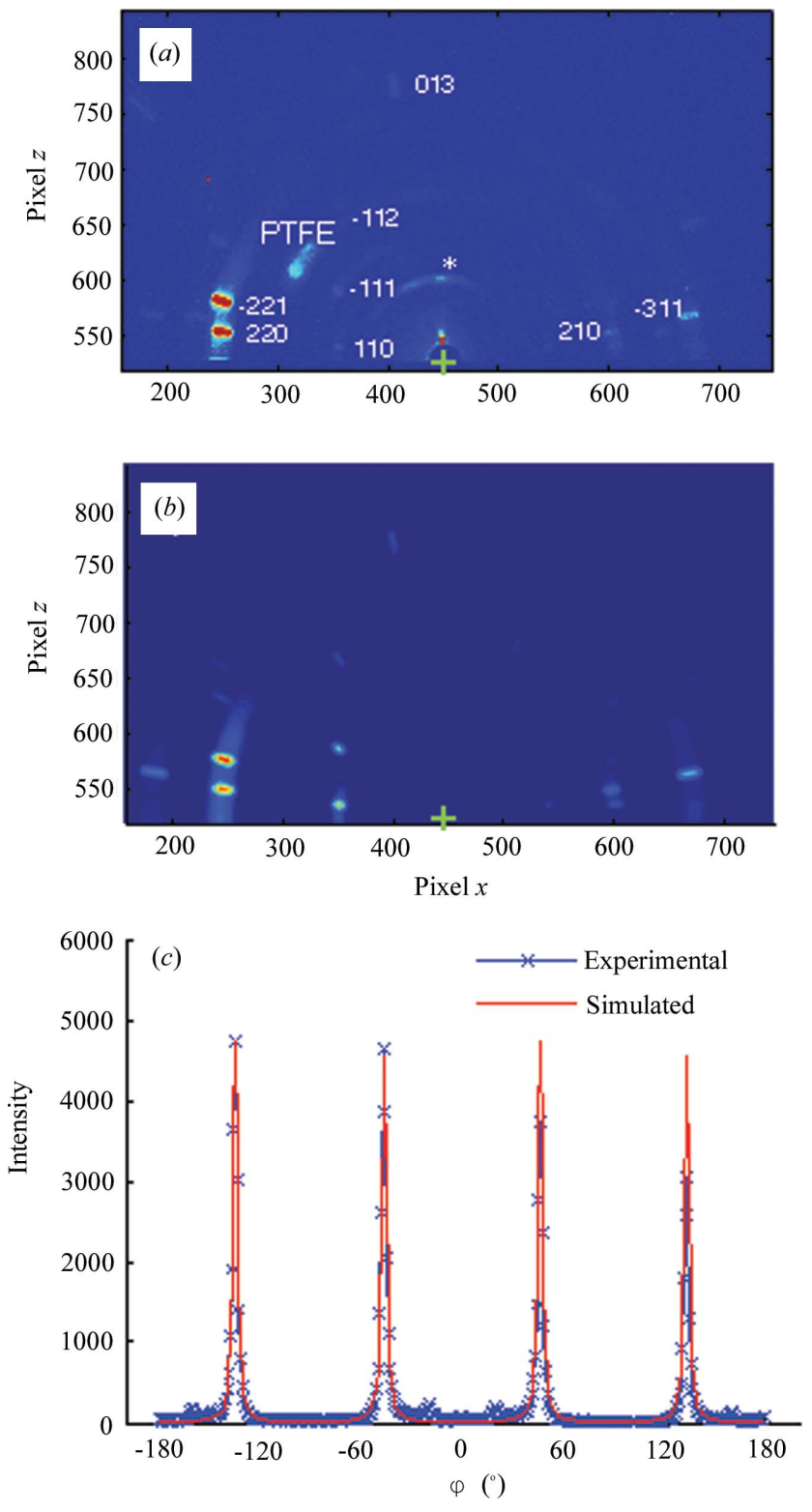

Figure 6

Comparison of $(a)$ the experimental diffraction image and $(b)$ the simulated image assuming highly biaxially oriented families of perylene crystallites having the $a b$ plane parallel to the substrate and the $\{110\}$ axes parallel to the PTFE alignment. The green '+' denotes the position of the direct beam. The radial peak broadening is accounted for discretely by calculating for different camera lengths $L$, varying from the nominal $120 \mathrm{~mm}$ by $\{-4,-3.75, \ldots, 4\} \mathrm{mm}$, thus corresponding to a beam footprint of $8 \mathrm{~mm}$ for this sample. The out-of-plane orientation distribution was modelled as the sum of two equally weighted fractions, one highly oriented (FWHM $\sim 1.8^{\circ}$ ) and one less oriented (FWHM $\sim 15^{\circ}$ ). The resulting reproduction of the perylene peak positions, widths and intensities is very good. The feature marked 'PTFE' corresponds to the PTFE 100 reflection, and the pseudo-specular scattering marked with an asterisk can be ascribed to the divergence of the incoming beam. (c) Synchrotron scan performed on a sample similar to that in $(a)$. This rocking scan about the sample normal was performed on the $\overline{2} 01$ reflection, revealing a high degree of in-plane orientation. The simulated curve was obtained assuming crystallites oriented with $\{110\}$ axes parallel to the PTFE alignment (see main text for further details). The smaller peaks in the scan are discussed by Breiby et al. (2008). 
oriented with an in-plane angle of $\sim 10^{\circ}$ between the PTFE alignment and the incoming beam (while keeping an incidence angle $\alpha_{\mathrm{i}} \simeq 0.2^{\circ}$ ). To improve the counting statistics, and to increase the number of Bragg peaks collected, the sample was made to oscillate by $\pm 4^{\circ}$ about the sample normal (angle $\varphi$ ) during the exposure time. This method is in fact very convenient for quickly establishing the presence of in-plane preferred orientations in thin films using conventional X-ray equipment. Furthermore, it can also give indications of the degree of preferred orientation, but clearly, without making assumptions, one image is insufficient to establish the full ODF. Therefore, a similar perylene on PTFE sample was measured at the synchrotron (Breiby et al., 2008), where Fig. $6(c)$ shows the resulting rocking scan obtained for a full rotation about the sample normal obtained with the (fixed position) point detector at $\left(Q_{x y}, Q_{z}\right)=(1.118,0.416) \AA^{-1}$, corresponding to the $\{201\}$ lattice planes.

As it turns out, the four principal peaks can be accounted for with a model having highly biaxially oriented crystallites with $\langle 110\rangle$ parallel to the PTFE alignment. The orientational distribution of crystallites was modelled by the ODF of equation (10), combined with two symmetry operations imposed by the alignment layer: For each crystallite orientation, there is (i) an equal probability of an orientation rotated by $180^{\circ}$ about the sample normal, and (ii) an equal probability of being mirrored about the PTFE alignment direction. In particular, from the synchrotron rocking scan in Fig. 6(c), but also from the high asymmetry of the image in Fig. 6(a), it is clear that the in-plane distribution is narrow. The synchrotron point detector scan was fitted by using $W_{\|}=3.2^{\circ}$ (FWHM), with $P_{3}$ in equation (12) given by a pseudo-Voigt function with relative weights 0.8 and 0.2 assigned to its Lorentzian and Gaussian parts, respectively. $P_{1}$ was taken as a pure Lorentzian, with width $W_{\mathrm{Q}}<0.03 \AA^{-1}$ (FWHM). The simulations were not very sensitive to $P_{2}$, which was taken to be Lorentzian with $W_{\perp} \simeq 1^{\circ}$ (FWHM).

For simulating the image in Fig. 6(b), the same model as described for the point detector scan was employed, but with a fixed incoming direction of the X-ray beam with respect to the PTFE alignment of $10^{\circ}$. To account for the continuous rocking of the sample during the experiment, an effective $W_{\|} \simeq 8^{\circ}$ was used. For this sample, weak scattering is observed in the $\omega_{\perp}$ direction near the Bragg peaks. The simulated image is therefore obtained as the equally weighted sum of two contributions: a highly oriented fraction with $W_{\perp} \simeq 1.8^{\circ}$ and a less oriented fraction with $W_{\perp} \simeq 15^{\circ}$. Scattering from PTFE was neglected in the simulations, but scattering from the (100) planes of PTFE at $Q=1.265 \AA^{-1}$ (Clark, 1999) can be seen in Fig. 6(a). This sample was larger than the perylene on $\mathrm{Si}$ sample, and a $8 \mathrm{~mm}$ footprint was employed. Further details of the perylene system will be published elsewhere (Breiby et al., 2008).

The computation time for producing Fig. 5(b) is less than $10 \mathrm{~s}$ on a Pentium $43.0 \mathrm{GHz}$ PC with 512 Mbyte RAM running Windows XP. Because of the heavier computation burden imposed by the symmetry operations in Fig. 6(b), the computation time for this image was $\sim 1 \mathrm{~min}$.

\section{Conclusion}

Based on the standard framework of kinematical X-ray diffraction, we have outlined a computationally efficient algorithm for simulating grazing-incidence X-ray diffraction from polycrystalline films. This algorithm is implemented in the computer program SimDiffraction. Particular attention has been given to the modelling of intensity for textured films with crystallites having an orientation distribution function tending towards cylindrical 'fibre' symmetry. Throughout, efforts have been made to keep the descriptions intuitively simple yet reasonably elegant. The use of the program was demonstrated for thin films of the organic material perylene. Being the first generally available program for simulating GIXD, we believe that SimDiffraction will be a valuable tool to the rapidly growing community using GIXD for material characterization.

We are grateful to Peter Hammershøj for generously contributing the perylene sample, to Robert Feidenhans'l for valuable discussions, and to the HASYLAB staff for technical assistance. This work was funded in part through a grant from the European Science Foundation through the Self-Organized Nanostructures (SONS) initiative (project 02-PE-SONS-130 NETSOMA).

\section{References}

Als-Nielsen, J., Jacquemain, D., Kjaer, K., Leveiller, F., Lahav, M. \& Leiserovitz, L. (1994). Phys. Rep. 246, 251-313.

Als-Nielsen, J. \& McMorrow, D. (2001). Elements of Modern X-ray Physics. New York: Wiley.

Breiby, D. W., Bunk, O., Pisula, W., Sølling, T. I., Tracz, A., Pakula, T., Müllen, K. \& Nielsen, M. M. (2005). J. Am. Chem. Soc. 127, 1128811293.

Breiby, D. W., Lemke, H. T., Hammershöj, P., Andreasen, J. W. \& Nielsen, M. M. (2008). J. Phys. Chem. C. In the press.

Breiby, D. W. \& Samuelsen, E. J. (2003). J. Polym. Sci. B Polym. Phys. 41, 2375-2393.

Bunk, O. \& Nielsen, M. M. (2004). J. Appl. Cryst. 37, 216-222.

Busch, P., Rauscher, M., Smilgies, D.-M., Posselt, D. \& Papadakis, C. M. (2006). J. Appl. Cryst. 39, 433-442.

Camerman, A. \& Trotter, J. (1964). Proc. R. Soc. London Ser. A, 279, 129.

Clark, E. S. (1999). Polymer, 40, 4659-4665.

Cromer, D. T. \& Mann, J. B. (1968). Acta Cryst. A24, 321-324.

Dosch, H. (1992). Critical Phenomena at Surfaces and Interfaces: Evanescent $X$-ray and Neutron Scattering. New York: Springer.

Evans-Lutterodt, K. W. \& Tang, M. T. (1995). J. Appl. Cryst. 28, 318326.

Feidenhans'l, R. (1989). Surf. Sci. Rep. 10, 105-188.

Gidalevitz, D., Feidenhans'l, R., Smilgies, D.-M. \& Leiserowitz, L. (1997). Surface Rev. Lett, 4, 721.

Kaganer, V., Moehwald, H. \& Dutta, P. (1999). Rev. Mod. Phys. 71, 779-819.

Kratky, O. (1933). Kolloid Z. 64, 213.

Kuzmenko, I., Rapaport, H., Kjaer, K., Als-Nielsen, J., Weissbuch, I., Lahav, M. \& Leiserowitz, L. (2001). Chem. Rev. 101, 1659-1696.

Lazzari, R. (2002). J. Appl. Cryst. 35, 406-421.

Lee, B., Park, I., Yoon, J., Park, S., Kim, J. Kim, K.-W., Chang, T. \& Ree, M. (2005). Macromolecules, 38, 4311-4323. 
Naudon, A. (1995). Modern Aspects of Small-Angle Scattering, edited by H. Brumberger, p. 191. Amsterdam: Kluwer Academic Publishers.

Renaud, G., Villette, B. \& Guenard, P. (1995). Nucl. Instrum. Methods Phys. Res. Sect. B, 95, 422.

Robinson, I. K. (1986). Phys. Rev. B, 33, 3830-3836.

Samuelsen, E. J., Aasmundtveit, K. E. \& Breiby, D. W. (2003). Electronic and Optical Properties of Conjugated Molecular Systems in Condensed Phases, edited by S. Hotta. Kerala: Research Signpost.

Sinha, S. K., Sirota, E. B., Garoff, S. \& Stanley, H. B. (1988). Phys. Rev. B, 38, 2297-2311.

Sirringhaus, H., Brown, P. J., Friend, R. H., Nielsen, M. M., Bechgaard, K., Langeveld-Voss, B. M. W., Spiering, A. J. H.,
Janssen, R. A. J., Meijer, E. W., Herwig, P. \& de Leeuw, D. M. (1999). Nature (London), 401, 685-688.

Smilgies, D. (2002). Rev. Sci. Instrum. 73, 1706-1710.

Smilgies, D.-M., Blasini, D. R., Hotta, S. \& Yanagi, H. (2005). J. Synchrotron Rad. 12, 807-811.

Toney, M. F. \& Brennan, S. (1989). Phys. Rev. B, 39, 7963-7966.

Vineyard, G. H. (1982). Phys. Rev. B, 26, 4146-4159.

Vlieg, E. (1997). J. Appl. Cryst. 30, 532-543.

Vlieg, E. (2000). J. Appl. Cryst. 33, 401-405.

Warren, B. E. (1969). X-ray Diffraction. New York: Dover Publications.

Wilson, A. J. C. (1995). International Tables for Crystallography, Vol. C. Dordrecht: Kluwer Academic Publishers.

Wittman, J. C. \& Smith, P. (1991). Nature (London), 352, 414-417. 